\title{
FUTURE ESTIMATION OF CONVENIENCE LIVING FACILITIES WITHDRAWAL DUE TO POPULATION DECLINE ALL OVER JAPAN FROM 2010 TO 2040 - FOCUS ON SUPERMARKETS, CONVENIENCE STORES AND DRUGSTORES -
}

\author{
Yuka Nishimoto $^{\mathrm{a} *}$, Yuki Akiyama ${ }^{\mathrm{b}}$, Ryosuke Shibasaki ${ }^{\mathrm{b}}$ \\ ${ }^{a}$ Graduate School of Frontier Science, The University of Tokyo, 156751@sbk.k.u-tokyo.ac.jp \\ ${ }^{\mathrm{b}}$ Center for Spatial Information Science, The University of Tokyo
}

Commission WG II/3

KEY WORDS: Future, Problem, Facilities, Urban, Data Mining, GIS

\begin{abstract}
:
Population explosion is considered to be one of the most crucial problems in the world. However, in Japan, the opposite problem: population decline has become serious now. Japanese population is estimated to decrease by twenty millions in 2040. This negative situation will cause to increase areas where many residents cannot make a daily living all over Japan because many convenience living facilities such as supermarkets, convenience stores and drugstores will be difficult to maintain their market area population due to future population decline.

In our research, we used point data of convenience living facilities developed by address geocoding of digital telephone directory and point data of future population projection developed by distribution of Japanese official population projection data proportionally among the building volume of digital residential map, which can monitor building volumes all over Japan.

In conclusion, we estimated that various convenience living facilities in Japan will shrink and close by population decline in near future. In particular, it is cleared that approximately $14.7 \%$ of supermarkets will be possible to withdraw all over Japan by 2040 . In addition, it is cleared that over $40 \%$ of supermarkets in some countryside prefectures will be possible to withdraw by 2040 . Thus, we estimated future distributions of convenience living facilities that cannot maintain their market area population due to future population decline. Moreover, we estimated the number of people that they will become inconvenience in buying fresh foods.
\end{abstract}

\section{INTRODUCTION}

Population explosion is considered to be one of the most crucial problems in the world. However, in Japan, the opposite problem: population decline has become serious now. Japanese population is estimated to decrease by twenty millions in 2040(Kaneko et al., 2008). Not only Japan, but also other developed countries such as Italy, Germany and South Korea have the same problem. Japan is one of the forefront countries of this problem. Japanese population is predicted to decline increasingly due to low birthrate and aging. This negative situation will cause to increase areas where many residents cannot make a daily living all over Japan because many convenience living facilities such as supermarkets, convenience stores and drugstores will be difficult to maintain their market area population due to future population decline. Actually, this negative impact already appeared in Japan(The Nikkei, 2016). Some Japanese peoples who live in rural areas and depopulated areas are becoming difficult to buy precooked fresh foods now. However, many previous studies in this field focused on limited areas in Japan. Moreover, researchers of previous studies did not have any other choice than using aggregated spatial data because of accessibility for disaggregated spatial data.

Therefore, it is a significant task to clear spatial distributions of such area in the future from a perspective of Japanese future national land policymaking.

Accordingly, the purpose of this research is estimation of future distributions of these facilities. Research target is supermarkets, convenience stores and drugstores that will be difficult to maintain their market area population due to future population decline from 2010 to 2040.

In our research, we used point data of convenience living facilities developed by address geocoding of digital telephone directory and point data of future population projection developed by distribution of Japanese official population projection data proportionally among the building volume of digital residential map, which can monitor building volumes all over Japan. Using these data, we estimated the number of residents within market area distance of each facility. Finally, we estimated whether these facilities satisfy the market area population or not from 2010 to 2040 . The market area distance in this research means the range to attract customers and the market area population means the number of people within the market area distance to maintain business.

\section{METHOD}

\subsection{Data}

In this study, we used following disaggregated data, in other words, point data.

- Point data of convenience living facilities

This data was developed by extracting the data of supermarkets, convenience stores and drugstores from a digital telephone book in 2010. In addition, estimated value of total floor space was integrated with these facilities by spatial integration of this data with digital residential map in 2009 . This process is necessary

\footnotetext{
* Corresponding author. 156751a@sbk.k.u-tokyo.ac.jp; Tel/Fax: +81 3-5452-(6417/6414)
} 
to decide the type of supermarket. Moreover, polygon data of urban area in 2011 (Ministry of Land, Infrastructure and Transport, 2011) was integrated with this data to divide the type of region for appropriate analysis. Finally, point data of convenience living facilities with attribute of total floor and inside-outside determination of urban area was developed.

- Point data of future population projection

This data is based on prediction data of future population(National Institute of Population and Social Security Research, 2010). Furthermore, the data of future population every $1 \mathrm{~km}$ square grid polygon from 2010 to 2040 is digitized. This data is based on future population projection every 5 years from 2010 to 2040 accumulated by $1 \mathrm{~km}$ square grid polygon (Hokkaido Intellect Tank). This study developed micro future population projection data by proportional distribution of the population projection according to total areas of each residential building by the residential map(Akiyama, 2015). However, the data in Fukushima prefecture is not included due to the accident of Fukushima Daiichi nuclear power plants in 2011. Figure1 shows the map of this point data.

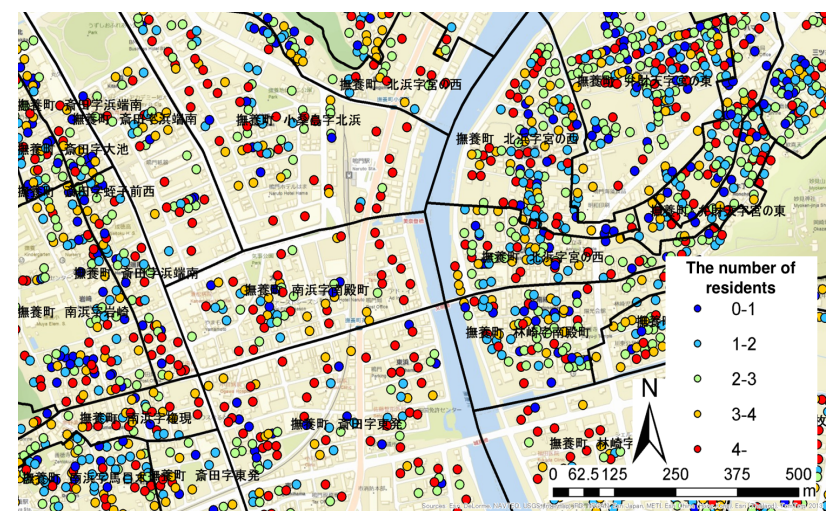

Figure 1. The map of point data of future population projection

\subsection{Analysis}

In the case of supermarkets, we classified all supermarkets into large type, medium type and small type on the basis of total floor space using table 1 . Second, we set the market area distance of table 2 using Euclidean distance. Then, we counted the number of residents within market area distance. Finally, we estimated whether supermarket satisfies the market area population or not from 2010 to 2040 using table 2 .

In the case of convenience stores, we estimated whether convenience store satisfies the market area population or not from 2010 to 2040 using table 3 and in the case of drugstores, using table4.

Table 1. A classification of supermarkets by total floor

\begin{tabular}{|c|c|c|c|c|}
\hline & Small & Medium & Large(GMS):Urban & Large(GMS):Suburb \\
\hline Market Area Distance[km] & 2 & 5 & 10 & 20 \\
\hline Market Area Population[people] & over 5,000 & over 10,000 & over 70,000 & over 100,000 \\
\hline
\end{tabular}

Table 2. Survival conditions of supermarkets

\begin{tabular}{|c|c|c|c|c|}
\hline & Small & Medium & Large(GMS):Urban & Large(GMS):Suburb \\
\hline Market Area Distance[km] & 2 & 5 & 10 & 20 \\
\hline Market Area Population[people] & over 5,000 & over 10,000 & over 70,000 & over 100,000 \\
\hline
\end{tabular}

Table 3. Survival conditions of convenience stores

\begin{tabular}{|c|c|}
\hline Market Area Distance[m] & 500 \\
\hline Market Area Population[people] & over 3,000 \\
\hline
\end{tabular}

Table 4. Survival conditions of drugstores

\begin{tabular}{|c|c|}
\hline Market Area Distance[km] & 3 \\
\hline Market Area Population[people] & over 20,000 \\
\hline
\end{tabular}

\subsection{Result}

Figure 2 and figure 3 show rates of supermarkets that satisfy their market area population in each municipality in 2010 and 2040. The result shows that the number of supermarkets with the possibility that cannot satisfy their market area population will keep increasing especially in prefectures and municipalities with depopulation. This trend will be remarkable in Kagoshima prefecture, Akita prefecture, Shimane prefecture, Nagasaki prefecture and Kochi prefecture. In particular, it is cleared that approximately $14.7 \%$ of supermarkets will be possible to withdraw all over Japan by 2040. In addition, it is cleared that over $40 \%$ of supermarkets in some countryside prefectures will be possible to withdraw by 2040. Additionally, figure 4 and figure 5 show supermarkets of some city in Japan in 2010 and 2040. Compared to 2010, the number of supermarkets that cannot satisfy their market area population will increase in 2040 In addition, figure 6 and figure 7 show rates of convenience stores that satisfy their market area population in each municipality in 2010 and 2040 . The result shows that the number of convenience stores with the possibility that cannot satisfy their market area population will keep increasing significantly all over Japan with depopulation. Compared to the result of supermarkets, the rates of convenience stores that cannot satisfy the survival conditions account for a large percentage.

Furthermore, figure 8 and figure 9 show rates of drugstores that satisfy their market area population in each municipality in 2010 and 2040. The result shows that the number of drugstores with the possibility that cannot satisfy their market area population will keep increasing with depopulation.

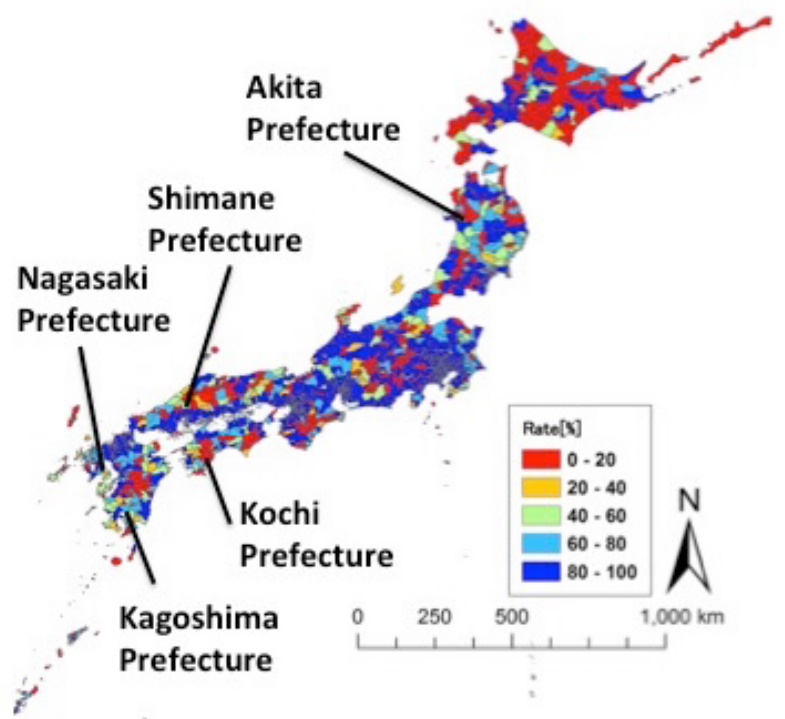

Figure 2. Rates of supermarkets that satisfy the survival conditions in 2010 


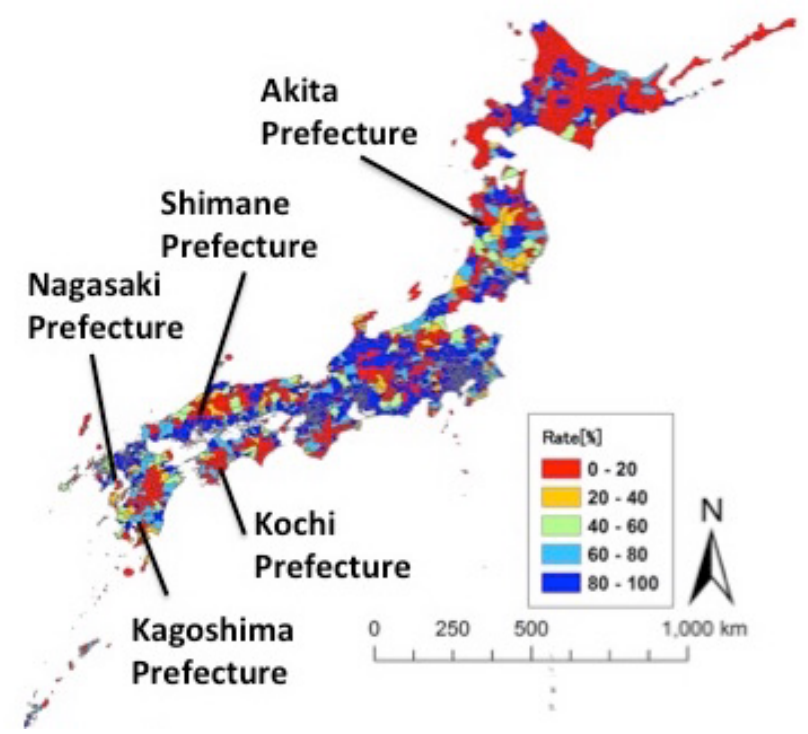

Figure 3. Rates of supermarkets that satisfy the survival conditions in 2040

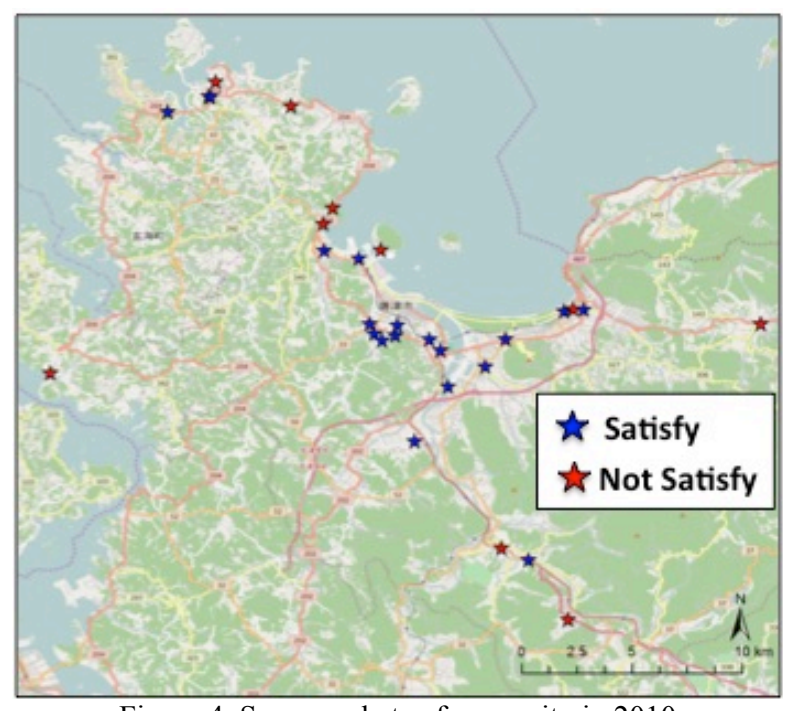

Figure 4. Supermarkets of some city in 2010

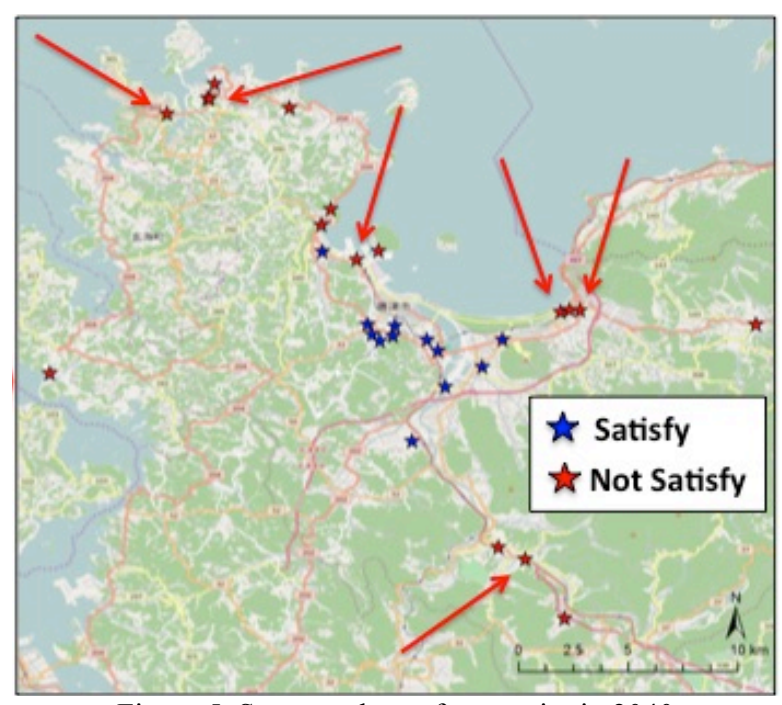

Figure 5. Supermarkets of some city in 2040

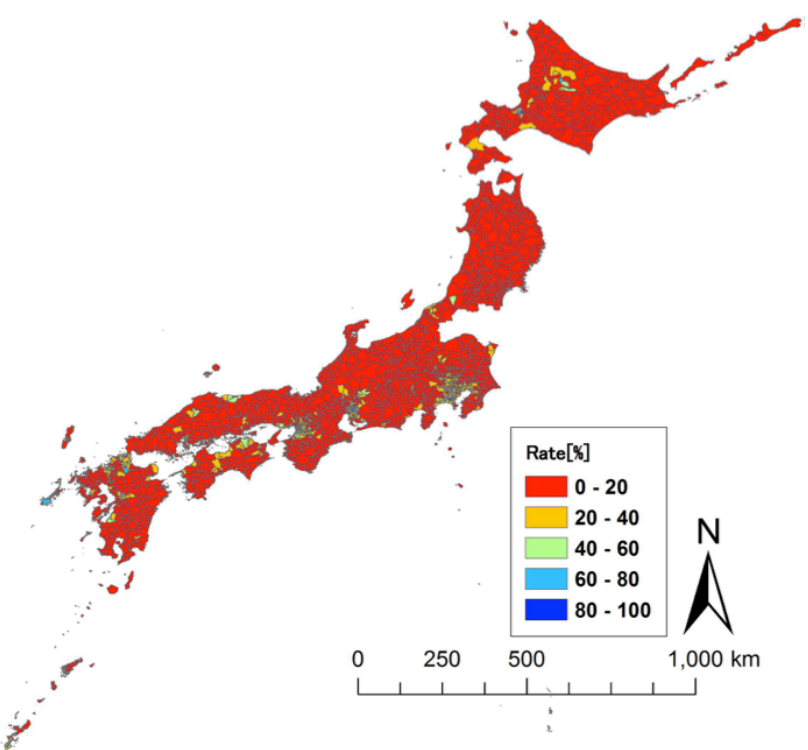

Figure 6. Rates of convenience stores that satisfy the survival conditions in 2010

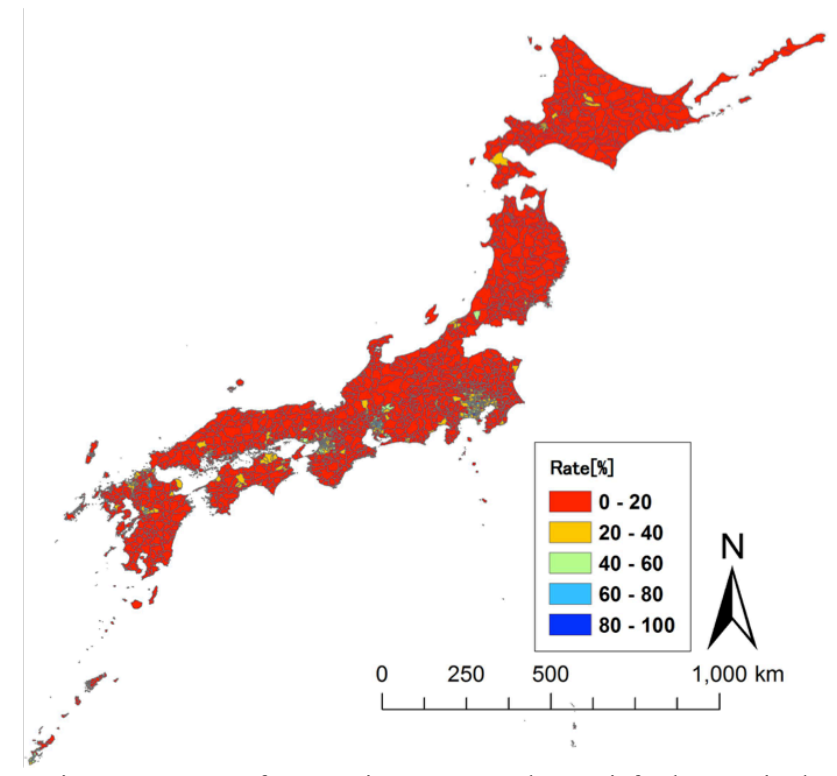

Figure 7. Rates of convenience stores that satisfy the survival conditions in 2040 


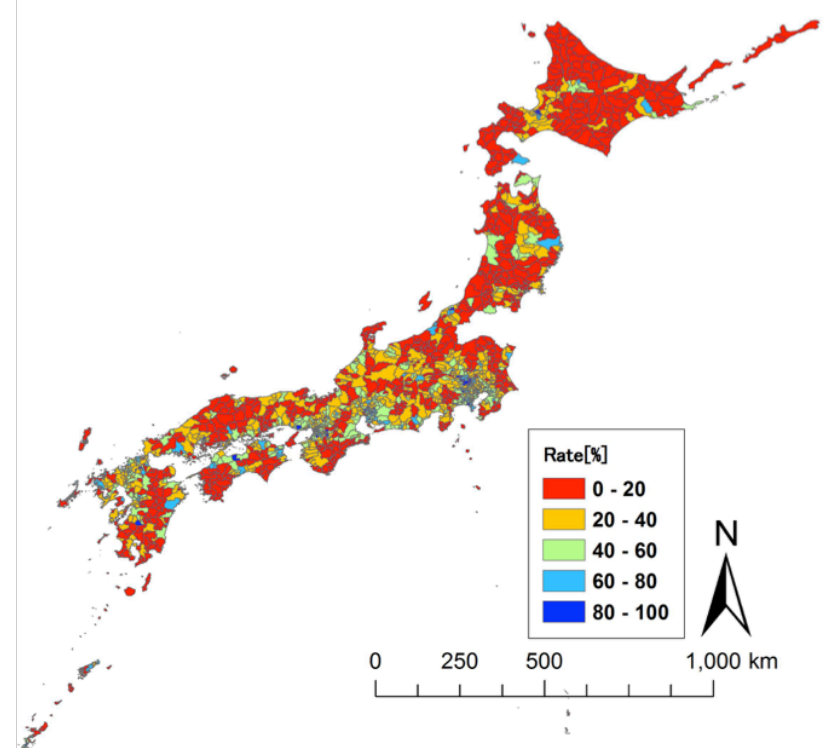

Figure 8 . Rates of drugstores that satisfy the survival conditions in 2010

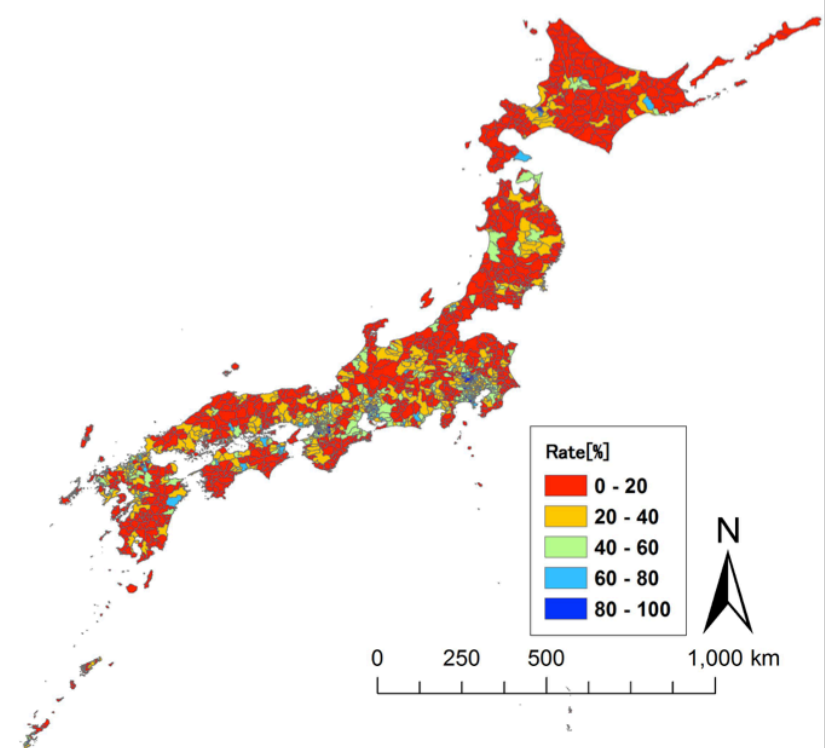

Figure 9. Rates of drugstores that satisfy the survival conditions in 2040

\section{CONCLUSIONS}

We estimated that various convenience living facilities in Japan will shrink and close by population decline in near future. In particular, it is cleared that approximately $14.7 \%$ of supermarkets will be possible to withdraw all over Japan by 2040. In addition, it is cleared that over $40 \%$ of supermarkets in some countryside prefectures will be possible to withdraw by 2040.Thus, we estimated future distributions of convenience living facilities that cannot maintain their market area population due to future population decline.

After this, we are going to use new method of analysis to analyze more closely. We plan to use gravity model. Therefore, we aim at making the case of fittest arrangement for Japanese future national land policymaking.

\section{ACKNOWLEDGEMENTS}

Authors wish to thank Hokkaido Intellect Tank (HIT) for provide the grid future population projection data. In addition, we would like to thank CSIS (the joint research No. 122) the University of Tokyo for their member's contributions.

\section{REFERENCES}

Kaneko, R., Ishikawa, A., Ishii, F., Sasai, T., Iwasawa, M., Mita, F. and Moriizumi, R., 2008. Population Projections for Japan: 2006-2055 Outline of Results, Methods, and Assumptions. The Japanese Journal of Population 2008, Vol.6, No.1

The Nikkei, 2016. The number of people who cannot buy daily necessities easily became 7 million people. (http://www.nikkei.com/article/DGXLASFS08H6M_Q6A130C1 $\mathrm{MM} 0000 /)$

Ministry of Land, Infrastructure and Transport, 2011. National Land Numerical Information.

Rebuild Japan Initiative Foundation., 2015. Population evaporation [the nation of 50 million people] shock of Japan. SINCHOSYA Publishing Co., Ltd.

National Institute of Population and Social Security Research, 2010

Akiyama, Y., 2015, "Future Estimation of Vacant House Distribution Using Micro Future Estimated Population Data", KAGIS Fall Conference 2015 \& International Symposium on GIS, 59-60.

Ministry of Economy, Trade and Industry, 2002. The Analysis of Commercial Environment.

Japanese Supermarket Association, 2014. Annual Investigation Report of Supermarkets. 